\title{
Risk-regulatory increase of trajectory safety, productivity and energy efficiency of motor transport operations in conditions of uncertainty
}

\author{
Arseniy Khabutdinov \\ State Enterprise "State Road Transport Research Institute", 57 Peremohy Ave, Kyiv, 03113, Ukraine
}

\begin{abstract}
The proposed method of complex increase of trajectory safety, productivity and energy efficiency of motor transport operations taking into account the laws of vehicle algorithmic control in difficult traffic conditions. Proceeding from the provisions of the theory of energy efficiency of a generalized type car and the laws of its adaptively-discrete energy of motion, the processes of functioning of the transport-ergatic systems "Driver-Vehicle" (TESDV) and control of the car using adaptive driving procedures (productive, safe, energy-efficient) in the conditions of information uncertainty of locally trajectory situations (LTS). In this work presents logic-procedural and mathematical models for the analysis of risk-regulatory algorithms for control of vehicle, taking into account the functioning of TESDV and quantitative assessment of operator-interface complexity of road sections. The basis of the method of estimating the information complexity of the operator-road interface in relation to any element of the road is the mathematical model of the entropy of the object (as a measure of disorder), which is used in technical cybernetics. For each section of the road, the total indicator of information complexity is determined, which takes into account three levels of uncertainty: low, medium and high. At medium and high levels of complexity of the road section, TESDV implements a risk-regulating automobile control algorithm. Proceeding from the essence of the regulatory labor procedures of driving, as well as their role in the formation of the modes of the TESDV, proposed results of operational simulation of algorithms regulation of locally-trajectory driving risks (LTR): counterproductive, sensory-tempo, incidental and anergic.
\end{abstract}

\section{Introduction}

Motor transport is a sphere of complex human-machine (ergatic) labor and energy-consuming transportation of goods and passengers in a conflict street-road network. Prior to motor transport operations (MTO) three requirements for their integrated performance are presented: "Productivity (P) - Trajectory safety (TS) Energy Efficiency (EE)". These requirements in the MTO provide a productive element of the motor transport - transport ergatic system "driver-vehicle" (TESDV) based on labor and machine procedures for the control of the vehicle.

In the said process, the first two requirements (P-TS) are controversial in the procedures for adjusting the speed of the vehicle. When alternating dangerous and safe sections of the road, which belong to the length of the mileage vehicle in the given MTO, the driver alternately uses safe and productive motivation for the control of the vehicle. This leads to the motivated formation of uneven-intermittent TESDV modes.

In terms of the ergatic functioning of TESDV, this motion is adaptively discrete and trajectory. As a result of this movement a trouble-free MTO is performed.
However, at the same time in the MTO is implemented a negative (in terms of technological cost-effectiveness of transportation) phenomenon of operational anergic of the vehicle (a phenomenon of simultaneous reduction of productivity and energy efficiency in the zones of locally-trajectory hazards (LTH) [1]. It is characterized by a significant and simultaneous decrease in the values of average speeds ( $1.5 \ldots 2.5$ times) and indicators of its energy efficiency (in $2 \ldots 4$ times). In this connection, ergatic bases of increase comprehensive success of motor transport operation are actual, which make it possible to improve the processes of TESDV's operation.

The methods of information analysis of the current speed of the car in the road traffic flow do not take into account the above three requirements for MTO, as well as the properties of the elements TEDSV and sections of the road [2].

\section{Main part}

At present, the following conditions are met for the fulfillment of certain requirements for the success of a MTO [3]. In particular, TESDV trajectory safety (TS) is carried out due to several factors:

* Corresponding author: habut20@gmail.com 
a) timely detection of visual signs of LTH;

b) estimation of the level of complexity of the site of the vehicle trajectory for the driver-operator or for the onboard information and control system of the vehicle;

c) prevention of the development of the LTH into the trajectory incident (TI) is an event of a shock intersection of the vehicle trajectory with another object; d) prevention of any possibility of realization of the mechanism of an emergency MTO (LTH $\rightarrow$ TI $\rightarrow$ Accident).

The demand for productivity (P) of MTO is also carried out on the basis of procedures of trajectoryadequate increase of the average speed of the vehicle in MTO in the absence of visual signs of the LTH. The MTO Energy Efficiency Requirement (EE) is met by rationally reducing the value of the car's anergic index with adaptively discrete TESDV movement in MTO, taking into account both TS and P requirements

These conditions of comprehensive performance of MOT must be carried out to the same extent as TESDV, as well as "unmanned" vehicle. It is established that taking into account the fuzzy level of information complexity of the site of the vehicle trajectory, TESDV can provide three levels of productivity of the MTO:

a) safe-successful MTO (SSTO, one requirement of TS);

b) functional-successful MTO (FSTO, two requirements$\mathrm{P}$ and TS); c) technologically-successful MTO (TSTO, three requirements $\mathrm{P}, \mathrm{TS}, \mathrm{EE}$ ).

In the implementation of these requirements, the driver can choose three types of adaptive vehicle control procedures: productive ( $\mathrm{P}$ - procedures), safe (TSprocedures) and energy-efficient (EE- procedures) [3,5]. Based on the above three principles of ergative successful control of vehicle are formed:

a) principle of distance estimation of the fuzzy level of information complexity of the section of the trajectory of the car's motion;

b) principle of remotely- advance choice of a behavioral installation and the purpose of the control of the vehicle in the form of a variant of the success of MTO (SSTO, FSTO, TSTO);

c) principle of remotely- advance choice of local tactics and algorithm of adaptive driving procedures.

These principles should be implemented in the methods of modernization improvement of the processes of vehicle control or "unmanned" car as a transport robotic tool, taking into account the properties of elements TESDV.

Within the framework of TESDV, the driver-operator in controlling a vehicle implements a number of properties: psychophysiological [3], labor-motivational and labor-procedural $[4,5]$. By labor-motivational belong to the ability of the driver trajectory-adequately choose behavioral settings and management objectives - in the form of options for levels of MTO (SSTO, FSTO, TSTO). Labor-procedural properties enable the driver to select and implement six driving procedures $P_{L i}(1)$ in accordance with the requirements of the complex success of the MTO $[3,5]$.

As an element of TESDV in the control process, the vehicle implements four groups of operational and technical properties of a car [6]: complex energy transformer machine, dangerous traffic control object, a vehicle, the source of machine procedures $P_{M i}$ of transportation. The plurality of labor procedures of the driver (PL) and the machine procedures of the vehicle $(\mathrm{PM})$ are as follows:

$$
\begin{gathered}
P_{L i} \in\left(P_{L i l}, P_{L i 2}, P_{L i 3}, P_{L i 4}, P_{L i j}, P_{L i 6}\right), \\
P_{M i} \in\left(P_{M i l}, P_{M i 2}, P_{M i 3}, P_{M i}, P_{M i 5}\right)
\end{gathered}
$$

where $P_{L i l}, P_{L i 2}, P_{L i 3}, P_{L i 4}, P_{L i \xi}, P_{L i 6}$ - labor procedures: sensory-perceptual, thought-analytical, sensory-tempo, predictive, solving, control, respectively; $P_{M i l}, P_{M i 2}, P_{M i 3}$, $P_{M i}, P_{M i 5}$ - machine procedures: energy-transforming, transmission, traction-coupling (or brake), trajectorykinetic (formation of kinetic energy of the vehicle in accordance with the given trajectory of motion), trajectory orientation (the choice of direction of movement along the road line ), respectively; $i$ - the index of the type of labor and machine procedures in their motivational orientation $(i=1$ - productive procedures, $i=2$ - anti-incident, $i=3$ - energy-efficient).

The process of driving a vehicle is divided into three stages: information-solving, motor and control. When operating TESDV in the first stage, the first four labor procedures of the driver $P_{L 1}, P_{L 2}, P_{L 3}, P_{L 4}$ are used. In the second stage - the labor procedure of the driver $P_{L 5}$, (with its four variants of actions on the management bodies of the vehicle). With the help of procedures $P_{L 5}$, the driver adjusts the intensity levels of the chain of machine procedures of the vehicle $P_{M}$. In the third stage, the driver, with the help of the procedure $P_{L 6}$, provides control of the parameters of the vehicle (speed, mileage, distance, acceleration, deceleration, etc.) in accordance with the objective of control.

An important initial task of the first stage of control a vehicle is the assessment of the fuzzy level of information complexity of the trajectory of the vehicle's for the driver. The method of solving this problem is presented below. The driver perceives information on trajectory elements of the road (with a traffic flow) in the section of the vehicle's traffic trajectory (SVTT), which are important for the qualitative performance of the first (information-solving) stage of traffic control. Two aspects of driver interaction with the road environment (operator-road interface) are important.

In the first aspect, the road is considered as an alternating rolling surface for a vehicle. This takes into account the state of the four factors of the road surface: the coefficient of adhesion of the wheels with the road, the angle of lifting the road, the roughness, the radius of the road curve. In the second aspect - as a conflict communicative channel for the adaptive movement of the vehicle. Eleven factors of this channel are considered: the number of lanes of the road; adjustable crossroads; unregulated crossroads; number of road signs; bus stop for public transport; public transport stops for drivers of other vehicles (not buses); SVTT in which the vehicle is moving at a constant speed; SVTT in which the vehicle is moving at an uneven velocity; unregulated ground pedestrian crossing; adjustable pedestrian crossing (ground); closed inspection area. The 
basis of the method for assessing the complexity of the information interaction of the driver with any element of the road (as the surface and the channel of movement) is a mathematical model of the entropy of the object (as a measure of disorder), which is used in technical cybernetics [7,8]. Entropy factor (disorderly measure) EN is defined as:

$$
E N=\kappa \cdot \ln (n)
$$

where $n$ - the number of possible (equally probable) states of the factor; $k$ - a constant value, taken $\kappa=1,5$. For $n=1$ we have $E N=0$, that is, at a single state of the factor, its entropy (disorderly measure) is zero. So, for $n$ $=10$ we have $E N=3,45$. The greater the number of states $n$, the higher the operator-interface complexity of this factor of the road in relation to the chain of three driver's labor procedures $\left(P_{L 1}-P_{L 2}-P_{L 3}\right)$ at the first stage of the vehicle control. In the methodological plan, the value of $E N_{i}$ for the $i$-th factor of the SVTT is equal to the magnitude of its operator-information complexity $P_{(O C) i}$, that is, $E N i=P_{(O C) i}$. These information models, which are based on the formalization of state sets of trajectory elements of the SVTT, are an important way of quantifying the level of complexity of the SVTT. The indicator of the total operator-interface complexity of a given section of the trajectory of the vehicle $P_{(O C)}(\Delta l)$ of length $\Delta l$, taking into account the foregoing, is defined as:

$$
P_{\mathrm{oc}}(\Delta l)=\sum_{i=1}^{4} P_{\mathrm{oc} i} \cdot n_{\mathrm{e} i}+\sum_{j=1}^{11} P_{\mathrm{oc} j} \cdot n_{\mathrm{e} j}
$$

where $i$ - index of factors of the operator-interface complexity of interaction with the road as with the rolling surface; $j$ - index of factors of operator-interface complexity of interaction with the road as a conflict communicative channel; $n_{e i}$ and $n_{e j}$ - the number of factors of the ${ }_{i}$-th and $j^{\text {-th }}$ species in the area of the trajectory of motion, respectively; $\Delta l$ is the length of the specified area of the vehicle's trajectory.

Fifteen mathematical models were developed for estimating the complexity of each type of trajectory elements that take into account their operational status. For example, if the value of coefficient of adhesion of wheels to the $\operatorname{road} \varphi=0,75$, then the value of the operator-interface complexity parameter $P_{(O C) i}=0$. For $\varphi$ $=0,4$, we have $P_{(O C) i}=2,25$. As an example, in table 1 shows the estimated values of operator-interface complexity parameters $P_{(O C) j}$ for various factors of the city bus route. It is assumed that each factor is detected once, that is, $n_{e i}=n_{e i}=1$.

Based on formula (2), 15 calculation models were formed for the entropy assessment of the complexity of the driver's information interaction with 4 factors of the road as a vehicle's rolling surface and with 11 factors of the road as a communicative channel with conflict points. When calculating the entropy complexity indicators of the first group of road factors, their maximum, minimum and specified values are taken into account. Then calculating the entropy complexity indicators of the second group of road factors, the number of conflict points of various types and the traffic management scheme are taken into account. In addition, when determining the total indicator of the informational complexity of a road section, the entropy values of the kinematics of adaptive-discrete vehicle motion are taken into account: constant speed $\mathrm{Vc}$, speed increment during acceleration of the vehicle $+\Delta \mathrm{V}$, decrease in speed when braking the vehicle $-\Delta \mathrm{V}$. Further, table 1 presents the calculated values of the information complexity indicators of some factors of the road as a communicative channel with conflicting points, as well as factors of the kinematics of vehicle movement $\mathrm{Vc}$ and $+\Delta \mathrm{V}$

Table 1. Estimated values of parameters of operator-interface complexity $P_{(O C) j}$ for the given values of factors of interaction

\begin{tabular}{|c|c|c|c|c|c|c|c|c|c|c|}
\hline $\mathrm{n}_{\mathrm{L}}$ & $\mathrm{n}_{\mathrm{RI}}$ & $\mathrm{n}_{\mathrm{UI}}$ & $\mathrm{n}_{\mathrm{RS}}$ & $\mathrm{n}_{\mathrm{S}}$ & $\mathrm{V}$ & $\Delta \mathrm{V}$ & $\mathrm{n}_{\mathrm{UPC}}$ & $\mathrm{n}_{\mathrm{APC}}$ & $\mathrm{n}_{\mathrm{ZCR}}$ & \\
\hline 3 & 1 & 1 & 3 & 1 & $\begin{array}{c}60 \\
\mathrm{~km} / \\
\mathrm{h}\end{array}$ & $\begin{array}{c}30 \\
\mathrm{~km} / \\
\mathrm{h}\end{array}$ & 1 & 1 & 1 & $\begin{array}{l}\text { Total } \\
P_{(O C) i}\end{array}$ \\
\hline 1,6 & 3,2 & 4,1 & 1,6 & 7,5 & 3,7 & 4,0 & 9,1 & 5,3 & 9,7 & 49,8 \\
\hline
\end{tabular}
with the road on the city bus route.

In table 1 the following notation is adopted: $n_{L}$ - the number of lanes of the road; $n_{R I^{-}}$the number of regulated intersections; $n_{U I}$ - the number of unregulated intersections; $n_{R S}$ - the number of road signs; $n_{S}$ - the number of public transport stops; $V$ - the constant speed of the vehicle in the road section; $n_{U P C}$ - the number of unregulated pedestrian crossings; $n_{A P C}$ - the number of adjustable pedestrian crossings; $n_{Z C R}$ - the number of zones of closed review. Conditions of uncertainty of the information state of the SVTT arise:

a) if the vehicle speed $V$ becomes greater than the permitted $V t$ by Traffic Rules, $(V>V t)$;

b) if the total value of the operator-interface complexity indicator $P_{o c} \Delta l$ corresponds to the average and higher levels $P_{o c}(\Delta l)>0,35 P_{o c(\max )}$;

c) if in the SVTT formed zones of closed review of different types, as well as the driver observes the implicit features of LTH, that is, at the value of the function of the $f_{v a}$ of the visual attribute, $\operatorname{LTH} f_{v a}=0,5$.

In conditions of medium and high uncertainty, the MTO's comprehensive success is provided on the basis of the algorithm of adaptive regulation of locallytrajectory risks (LTR) of vehicle control [7]. Based on the essence of the processes of the TESDV, as well as the modes of adaptive-discrete motion of the vehicle, four models for evaluation and regulation of the LTR are proposed. These models are as follows:

$$
\begin{gathered}
r_{c p}=1-V / V_{t}, r_{c p} \rightarrow \text { min, } r_{c p} \in(0 ; 1), f_{v s}=0 \\
r_{s t}=a_{1} \cdot V / V_{t}, r_{s t} \rightarrow \text { min, } r_{s t} \in(0 ; 1), f_{v s}=0,5 \\
r_{i}=\Delta V_{a} / V, r_{i} \rightarrow \text { min, LTR } \rightarrow \text { invers, } r_{i} \in(0 ; 1), f_{v s}=1 \\
r_{a}=1-K_{a c} / K_{a o}, r_{a} \rightarrow \text { min, } r_{a} \in(0 ; 0,8), f_{v s}=(0 ; 0,5)
\end{gathered}
$$

where $r_{c p}, r_{s t}, r_{i}, r_{a}$ - the values of the parameters of LTR: counterproductive, sensory-tempo, incident and anergic, respectively; $V$ - current speed of the vehicle; $V_{t}$ - value of speed, regulated by Traffic Rules; $f_{v s}$ - functional of 
the visual signs of the LTH; $\Delta V_{a}$ - anti-incident reduction of the speed of the vehicle, which ensures that the trajectory incident (TI) is prevented; $K_{a c}$ and $K_{a o}$ constructive and operational indicators of car anergicity values; $a_{1}$ - constant dimensionless quantity; "Invers» operator of anti-incident inversion of LTH in safety.

Two variants of algorithmic control of a vehicle are developed under the condition of procedural ensuring of the complex success of the MTO taking into account the levels of complexity (uncertainty) of the SVTT. First, at low levels of uncertainty of the information state of the SVTT $\left(P_{o c}(\Delta l)<0,35 P_{o c(\max )}\right)$, the principle of adapting the modes of the vehicle to four types of locally trajectory situations (LTS): SLTS (safe), PDLTS (predangerous), DLTS (dangerous) and CLTS (critical).

Proceeding from this principle, four variants of situational algorithms of vehicle control and adaptive tactics vehicle speed control (productive, anti-incident and energyefficient) have been formed. At medium and high levels of uncertainty of the information state of the SVTT $\left(P_{o c}(\Delta l)<\right.$ $\left.0,35 P_{o c(\max )}\right)$, the principle of adapting the modes of vehicle movement to four types of LTR is used (see Expressions 47). Proceeding from this principle, three variants of riskregulatory algorithms of vehicle control and adaptive tactics vehicle speed control (productive, anti-incident and energyefficient) are formed. For the quantitative assessment of the quality of algorithmic control of the vehicle in multiphase MTO with anti-incidence phases, designed calculation schemes and mathematical models for analyzing the dimensionless indices of: average speed in MTO- $K_{V}$, the energy consumption of the vehicle $K_{E}$, the anergic of the vehicle $K_{A}$ in the test MTO $[5,6]$ :

$$
\begin{gathered}
K_{p}=V_{A} / V_{R S}, K_{p} \in(0,5 ; 1,5, \\
K_{E}=E_{F} / E_{R S}, K_{E} \in(1,15 ; 4), \\
K_{A}=K_{E} / K_{P V}, K_{A} \in(1,0 ; 6),
\end{gathered}
$$

where $V_{A}$ and $V_{R S}$ - average speed of the vehicle in the test operation and the value of the reference speed of the vehicle $\left(V_{R S}=11,1 \mathrm{~m} / \mathrm{s}\right) ; E_{F}$ - energy consumption of the forces of resistance motion of the vehicle in the typical test operation $(J) ; E_{R S}$ - energy consumption for moving the calculated reference vehicle at constant speed $V_{R S}$.

TESDV movement is carried out in multiphase MTO. In the general case, the structure of MTO includes four typical phases: a) VT - phase, V = const; b) STphase, "car acceleration " with $\Delta \mathrm{V}>0$; c) ZT- phase, " car braking " with $\Delta \mathrm{V}<0$; d) ZTn-phase, "car rolling", $\Delta \mathrm{V}<0$ in the absence of motor driver actions $P_{L i 4}=P_{L i 5}$ $=0$. As a starting expression for the formation of a techno-ergatic model of speed control of the car (according to the technical scheme) in multiphase MTO, the law of change of the kinetic energy of the car is known in Car Theory [9]:

$$
\frac{d \mathrm{E}_{\mathrm{ki}}}{d t}=N_{\mathrm{lpi}}(t)-N_{1 \mathrm{i}}(t),
$$

where: $E_{k i}$ cinetic energy of the car; $\mathrm{N}_{1 \mathrm{pi}}$ - power of the internal forces of the car, $p=1,2,3(p=1$ - corresponds to the effective power of the engine of the car at the phases VT and ST in MTO; $p=2$ - corresponds to the brake power of the engine at the compression modes of forced braking of the motor in phases ZT- "roll-up"; p = 3 - corresponds to the power of friction forces in the brake mechanisms of the car in the phases of "braking" $\mathrm{ZT}$ ); $\mathrm{N}_{21}$-the power of the resistance to the movement of the vehicle; i - index of phase number of MTO.

On the basis of (11) the general technical-ergatic model of regulation of speed of the car (as a complex machine) in the phases of transport operation is obtained [6]:

$$
\begin{aligned}
& \mathrm{V}\left(\mathrm{P}_{\mathrm{Li} 5}, \mathrm{~K}_{\mathrm{jr}}, \mathrm{D}_{\mathrm{rs}}, \mathrm{D}_{\mathrm{cc}}, \mathrm{t}\right)=\mathrm{A}_{1}\left(\mathrm{P}_{\mathrm{Li} 5}, \mathrm{~K}_{\mathrm{jr}}, \mathrm{D}_{\mathrm{rs}}, \mathrm{D}_{\mathrm{cc}}, \mathrm{t}\right)+ \\
& +\mathrm{A}_{2}\left(\mathrm{P}_{\mathrm{Li} 5}, \mathrm{~K}_{\mathrm{jr}}, \mathrm{D}_{\mathrm{rs}}, \mathrm{D}_{\mathrm{cc}}, \mathrm{t}\right) \times \exp \left(\mathrm{B}\left(\mathrm{P}_{\mathrm{Li} 5}, \mathrm{~K}_{\mathrm{jr}}\right) \times \mathrm{t}\right)
\end{aligned}
$$

where: $A_{1}, A_{2}, B$ - are the parameters of the model of the generalized car velocity, which are functions of the sets of characteristics: labor motor procedures of the driver$\mathrm{P}_{\mathrm{Li} 5}$; of the set of Car Generalized Type Designs - $\mathrm{K}_{\mathrm{jr}}$; sets of road properties as rolling surface $-D_{r s}$ and communication channel - $\mathrm{D}_{\mathrm{cc}}$; t- duration of the i-th phase of MTO; $j$ - car class index by load capacity; $r$ - is the index of the version of car designs in the $j$-th class.

Taking into account (11) and (12), the regularities of the kinematics of the controlled motion of the car at different phases of MTO (VT, ST, ZT) are obtained: velocities- $\mathrm{V}_{\mathrm{v}}\left(\mathrm{P}_{\mathrm{Li} 5}\right), \mathrm{V}_{\mathrm{s}}\left(\mathrm{P}_{\mathrm{Li} 5}, \mathrm{t}\right), \mathrm{V}_{\mathrm{z}}\left(\mathrm{P}_{\mathrm{Li} 5}, \mathrm{t}\right)$; the paths of motion are $-1_{v}\left(P_{L i 5}\right), 1_{s}\left(P_{L i 5}, t\right), 1_{z}\left(P_{L i 5}, t\right)$. Techno-ergatic models of energy of controlled motion of a car (according to the technological scheme of action of complex machine and source of machine procedures) were obtained, taking into account labor $\mathrm{P}_{\mathrm{L}}$ and machine $\mathrm{P}_{\mathrm{M}}$ procedures of TESDC in the analysis of energy consumption $\mathrm{E}_{\mathrm{v}}\left(\mathrm{V}_{\mathrm{v}}\right)$ and fuel consumption $Q_{v}\left(V_{v}, T_{v}\right)$ then:

$$
\begin{aligned}
& \quad E_{\mathrm{v}}\left(V_{\mathrm{v}}\right)=l_{\mathrm{v}} \cdot\left(A_{3}+A_{4} \cdot V_{\mathrm{v}}+A_{5} \cdot V_{\mathrm{v}}^{2}+\right. \\
& \left.\quad+A_{6} \cdot V_{\mathrm{v}}^{3}+A_{7} \cdot V_{\mathrm{v}}^{4}+A_{8} \cdot V^{5}\right) \\
& Q_{\mathrm{v}}\left(V_{\mathrm{v}}, t_{\mathrm{v}}\right)=t_{\mathrm{v}} \cdot\left(B_{4}+B_{5} \cdot V_{\mathrm{v}}+B_{6} \cdot V_{\mathrm{v}}^{2}+\right. \\
& \left.+B_{7} \cdot V_{\mathrm{v}}^{3}+B_{8} \cdot V_{\mathrm{v}}^{4}+B_{9} \cdot V^{5}\right)
\end{aligned}
$$

where: $A_{3}, A_{4} \ldots A_{8}, B_{4}, B_{5}, B_{6}, \ldots . B_{9}$ - constant parameters, which depend on the technical parameters of the car, the characteristics of the road, the parameters of the $h_{e v}$ engine control and the gearbox $u_{g v}$ in the VT phases of MTO.

The energy of the forces of resistance to motion of the car in ST-phases (acceleration) is determined as follows:

$$
\begin{aligned}
& \mathrm{E}_{\mathrm{s}}\left(\mathrm{t}, \Delta \mathrm{V}_{\mathrm{s}}\right)=\mathrm{P}_{\mathrm{cs}}(\mathrm{t}) \cdot \mathrm{1}_{\mathrm{s}}\left(\mathrm{t}_{\mathrm{s}}\right)=\mathrm{B}_{10} \Delta \mathrm{V}_{\mathrm{s}}+\mathrm{B}_{11}\left(\Delta \mathrm{V}_{\mathrm{s}}\right)^{2}+ \\
& +\mathrm{B}_{12}\left(\Delta \mathrm{V}_{\mathrm{s}}\right)^{3}+\mathrm{B}_{13} \cdot \mathrm{t}_{\mathrm{s}}+\mathrm{B}_{14} \cdot \mathrm{t}_{\mathrm{s}} \cdot \Delta \mathrm{V}_{\mathrm{s}}+\mathrm{B}_{15} \cdot \mathrm{t}_{\mathrm{s}} \cdot \Delta \mathrm{V}_{\mathrm{s}}^{2}
\end{aligned}
$$

where: $\mathrm{B}_{16}, \mathrm{~B}_{17}, \mathrm{~B}_{18}, \mathrm{~B}_{19}, \mathrm{~B}_{20}, \mathrm{~B}_{21}$ - constant parameters depending on the set of constructive technical parameters $\mathrm{K}_{\mathrm{jr}}$ and control parameters $\mathrm{h}_{\mathrm{es}}$ and $\mathrm{u}_{\mathrm{gsv}} ; \mathrm{t}_{\mathrm{s}}-$ during the ST (acceleration) phase. 
Also obtained the following model of fuel consumption in ST-phases:

$$
\begin{aligned}
& \mathrm{Q}_{\mathrm{s}}\left(\mathrm{t}, \Delta \mathrm{V}_{\mathrm{s}}\right)=\mathrm{B}_{16} \cdot \mathrm{t}_{\mathrm{s}}+\mathrm{B}_{17} \cdot \Delta \mathrm{V}_{\mathrm{s}}+\mathrm{B}_{18} \cdot\left(\Delta \mathrm{V}_{\mathrm{s}}\right)^{2}+ \\
& +\mathrm{B}_{19} \cdot\left(\Delta \mathrm{V}_{\mathrm{s}}\right)^{3}+\mathrm{B}_{20} \cdot \mathrm{t}_{\mathrm{s}} \cdot \Delta \mathrm{V}_{\mathrm{s}}+\mathrm{B}_{21} \cdot \mathrm{t}_{\mathrm{s}} \cdot \Delta \mathrm{V}_{\mathrm{s}}^{2}
\end{aligned}
$$

where $\mathrm{B}_{16}, \mathrm{~B}_{17}, \mathrm{~B}_{18}, \mathrm{~B}_{19}, \mathrm{~B}_{20}, \mathrm{~B}_{21}-$ are constant parameters that depend on the set of structural and technical parameters $\mathrm{K}_{\mathrm{jr}}$, the control parameters of the car $\mathrm{h}_{\mathrm{es}}$ and $\mathrm{u}_{\mathrm{gs}}$.

The magnitude of the coefficient of a car speed $K_{v}$ in a typical test operation is defined as:

$$
\mathrm{K}_{\mathrm{v}}=\mathrm{V}_{\mathrm{c}} / \mathrm{V}_{\mathrm{cs}}, \mathrm{K}_{\mathrm{v}} \in(0,5 ; 1,5)
$$

where $\mathrm{V}_{\mathrm{c}}$ - is the average velocities of the car in test transport operation; $\mathrm{V}_{\mathrm{cs}}$ - is constant vehicle speed in a standart transport operation, in this case $\mathrm{V}_{\mathrm{cs}}=11.1 \mathrm{~m} / \mathrm{s}$.

The values of energy and fuel coefficients $\mathrm{K}_{\mathrm{e}}$ and $\mathrm{K}_{\mathrm{q}}$ in a test operation are defined as the ratio:

$$
\begin{gathered}
\mathrm{K}_{\mathrm{e}}=\mathrm{E}_{0} / \mathrm{E}_{\mathrm{s} 0}, \mathrm{~K}_{\mathrm{e}} \in(1,15 ; 4), \\
\mathrm{K}_{\mathrm{q}}=\mathrm{Q}_{0} / \mathrm{Q}_{\mathrm{s} 0}, \mathrm{~K}_{\mathrm{q}} \in(1,1 ; 3)
\end{gathered}
$$

where $\mathrm{E}_{\mathrm{o}}-$ is the total energy consumption of the resistance forces of the car motion in a test operation (joule ); $\mathrm{E}_{\mathrm{so}}$ energy consumption for moving a car in a standart transport operation at speed V $=11,1 \mathrm{~m} / \mathrm{s}$; Qois the total fuel consumption of the car in a test operation (grams or liters / km); $\mathrm{Q}_{\mathrm{so}}-$ is the fuel consumption of the reference car at a speed of $\mathrm{V}=11.1 \mathrm{~m} / \mathrm{s}$ in a standart transport operation.

The energy-efficient tactics of control of the car in the test MTO is realized by minimizing the index of its operational anergicity (the phenomenon of operational decrease in the performance and energy efficiency of the car in adaptive transport operations in the presence of locally trajectory hazards):

$$
\mathrm{K}_{\mathrm{a}}=\frac{\mathrm{K}_{\mathrm{e}}}{\mathrm{K}_{\mathrm{v}}}=\frac{\mathrm{K}_{\mathrm{e}}\left(\mathrm{K}_{\mathrm{v}}, \mathrm{K}_{j \mathrm{r}}, \mathrm{Dr}, \mathrm{P}_{\mathrm{M}}\right)}{\mathrm{K}_{\mathrm{v}}\left(\mathrm{K}_{j \mathrm{r}}, \mathrm{P}_{\mathrm{L} 5}, \mathrm{f}_{\mathrm{vs}}, \mathrm{d}_{\mathrm{LTH}}, \mathrm{Dr}, \mathrm{P}_{\mathrm{M}}\right)} \rightarrow \min
$$

where $\mathrm{P}_{\mathrm{m}}$ - machine driving procedures; $\mathrm{P}_{\mathrm{L} 5}$ - a subset of labor motor driving procedures; $\mathrm{f}_{\mathrm{vs}}$-visual sign of locally trajectory hazard (LTH); $\mathrm{d}_{\mathrm{LTH}}$-long distance to the LTH zone; ; $\mathrm{D}_{\mathrm{r}}$-set of road characteristics.

The mathematical construction of the model of the index of its operational fuel anergicity of the car $\mathrm{K}_{\mathrm{aq}}, \mathrm{K}_{\mathrm{aq}}$ $=\mathrm{K}_{\mathrm{q}} / \mathrm{K}_{\mathrm{v}}$ has a similar form.

The dimensionless values of the energy efficiency coefficients $-\mathrm{P}_{\mathrm{e}}$ and the fuel efficiency $-\mathrm{P}_{\mathrm{q}}$ of the car are defined as the inverse values of the indicators of operational anergicity- $\mathrm{K}_{\mathrm{a}}$ and $\mathrm{K}_{\mathrm{aq}}$ energy indices:

$$
\mathrm{P}_{\mathrm{e}}=1 / \mathrm{K}_{\mathrm{a}}, \mathrm{P}_{\mathrm{q}}=1 / \mathrm{K}_{\mathrm{aq}} .
$$

On the basis of formulas (13-18) and logical-procedural schemes of TESDV functioning, software and algorithms for multivariate modelling of the functioning processes of the ergatic 'Driver-Bus' system in test operations have been developed. They allow you to analyse a complex of dimensionless energy indicators TESDV taking into account changes in various factors of this system (structural,

\begin{tabular}{|c|c|c|c|c|c|c|}
\hline \multirow{2}{*}{$\begin{array}{l}\text { Dimension- } \\
\text { less energy } \\
\text { efficiency } \\
\text { indicators } \\
\text { of the bus }\end{array}$} & \multicolumn{6}{|c|}{ Length of run between stops $\ln , \mathrm{m}$} \\
\hline & 100 & 200 & 400 & 600 & 800 & 1000 \\
\hline $\mathrm{K}_{\mathrm{E}}$ & 4,69 & 4,1 & 3,79 & 3,35 & 3,08 & 2,9 \\
\hline $\mathrm{K}_{\mathrm{A}}$ & 9,44 & 6,48 & 4,66 & 3,63 & 3,11 & 2,8 \\
\hline$K_{\mathrm{ns}}$ & 0,77 & 0,82 & 6,84 & 0,04 & 0,51 & 0,43 \\
\hline $\mathrm{K}_{\mathrm{L}}$ & 0,49 & 0,63 & 0,81 & 0,92 & 0,99 & 1,03 \\
\hline$\Pi_{\mathrm{E}}$ & 0,05 & 0,09 & 0,17 & 0,25 & 0,31 & 0,37 \\
\hline$\Pi_{\mathrm{Eq}}$ & 0,14 & 0,18 & 0,23 & 0,30 & 0,35 & 0,39 \\
\hline
\end{tabular}
technical, road, transport and operational). As an example, table 2 presents data for analysing the effect of the distance between bus stops on the values of the set of energy efficiency indicators for a bus.

Table 2. Results of the analysis of the bus operation "Bogdan A092" between the stops at different lengths of the run

were $K_{E}$ - energy consumption ratio, $K_{A}$ - indicator of vehicle's energy, $K_{n s}$ - the share of non-sustained modes in the operation, $K_{L}$ - local productivity factor , $\Pi_{E}-$ energy efficiency indicator, $\Pi_{E q}-$ fuel efficiency indicator.

Based on the theory of energy efficiency of cars of the generalized type [5], mathematical models were obtained for the determination and parametric analysis of the parameters of $K_{P}, K_{E}$ and $K_{A}$. On their basis, the task of multivariate analysis of the influence of a number of factors (constructive-technical, labor procedures, road and operational) is solved. In table 2 and fig. 1 presents some of the results of the analysis.

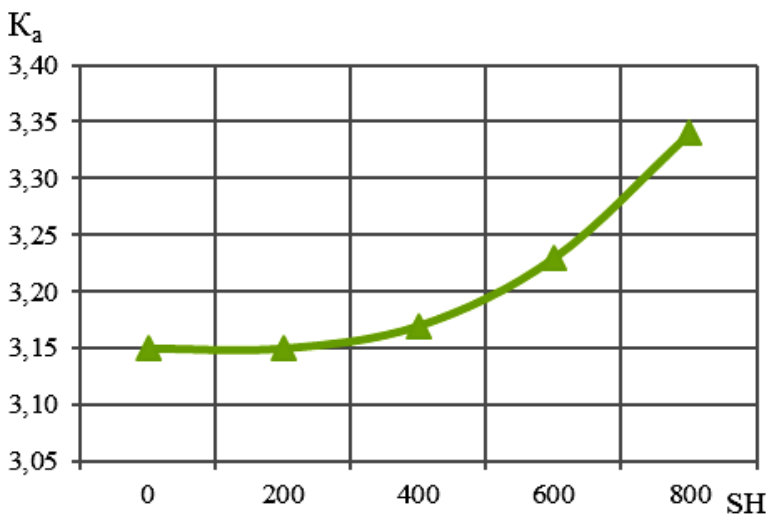

Fig. 1. Dependence of the indicator of the anergic $K_{A}$ of the bus "Bogdan A092" on the value of the road's roughness index $S H$ $(\mathrm{cm} / \mathrm{km})$

\section{Conclusions}

1. On the basis of the entropy model of technical cybernetics, a method of quantitative estimation of the information complexity of a section of the road was 
developed taking into account the diversity of states of the trajectory elements of the road and the traffic flow; it allows the driver-operator or on-board vehicle trajectory identification system to evaluate fuzzy (low, medium, high) levels of uncertainty of the trajectory section of the road to select situational (at low level) or risk- regulatory (at medium and high levels) car control algorithms.

2. It has been established that in order to comprehensively increase the productivity, trajectory safety and energy efficiency of transport operations in conditions of uncertainty, the driver should provide remote (according to visual signs of locally trajectory hazards) and preventive minimization of four types of locally trajectory driving risks: counterproductive, sensory-tempo (the possibility of deterioration in the perception of the driver of the road environment due to the high speed of the car), incident and anergic (the possibility of reducing the performance and energy efficiency of the car in difficult driving conditions).

3 . Using design schemes of the theory of adaptivediscrete motion of a generalized type car, techno-ergatic models of controlled kinematics and energy of a car in multiphase transport operations have been developed; based on them, mathematical and software of procedural-simulation modeling of the functioning of the transport ergatic system "driver-vehicle" (TESDV) was developed.

4. Based on the results of a multivariate and testsimulation analysis of the functioning of the ergatic driver-bus system, a complex of dimensionless performance indicators and transport energy efficiency of cars in procedurally safe transport operations is proposed; these indicators ensure the development of two relevant methods in Motor transport: safe and energy-efficient driving, as well as the technologically successful car operation in accordance with the requirements of a comprehensive increase in adaptive performance, trajectory safety and energy efficiency of the road transportations.

\section{References}

1. A. Khabutdinov, Visnyk NTU, 1, 519, (2015)

2. N. Kulbashnaya, N., Soroka, K. (2016). Development of a model for choosing the driver's speed with regard to the road environment. East European Journal of Advanced Technology, $3 / 2$ (81), 22-29. DOI: 10.15587/1729-4061.2016.71489. (Scopus)

3. O. Petrashevskiy, A. Khabutdinov, Transport problems, 6, 60, (2009)

4. V. Ilarionov, M. Koshelev, V. Mishurin, Driver and the car, (1985)

5. A. Khabutdinov, Project Management, Systems Analysis and Logistics, 8, 204, (2011)

6. R. Khabutdinov, O. Kocjuk, Energy-resource efficiency of the car, (1997)

7. R. Sapozhnikov, Fundamentals of technical cybernetics, (1970)

8. A. Khabutdinov, Transport problems, 7, 151, (2010)

9. A. Litvinov, Y. Farobin, Automobile: Theory of operational properties, (1989) 